\title{
Diagnostic accuracy of myocardial perfusion imaging in patients evaluated for kidney transplantation: A systematic review and meta- analysis
}

Jeroen R. Kelderman, BSc, ${ }^{a}$ Floris E. J. Jolink, BSc, ${ }^{\mathrm{b}}$ Stan Benjamens, MD, PhD, ${ }^{\mathrm{a}, \mathrm{b}}$ Andrea G. Monroy-Gonzalez, MD, PhD, ${ }^{a}$ Robert A. Pol, MD, PhD, ${ }^{\text {b }}$ and

Riemer H. J. A. Slart, $M D, P^{a, c}$

a University of Groningen, Medical Imaging Centre, Department of Nuclear Medicine and Molecular Imaging, University Medical Center Groningen, Groningen, The Netherlands

b University of Groningen, Division of Transplant Surgery, Department of Surgery, University Medical Center Groningen, Groningen, The Netherlands

c Department of Biomedical Photonic Imaging, Faculty of Science and Technology, University of Twente, Enschede, The Netherlands

Received Jan 28, 2021; accepted Mar 29, 2021

doi: 10.1007/s12350-021-02621-x

Background. Cardiovascular disease is the most common cause of death after kidney transplantation. Coronary artery disease (CAD) assessment is therefore mandatory in patients evaluated for transplantation. We aimed to assess the diagnostic accuracy for CAD of singlephoton emission computed tomography (SPECT) compared to the standards invasive coronary angiography (ICA) and coronary computed tomography angiography (CCTA) in patients evaluated for kidney transplantation.

Methods. We performed a systematic literature search in PubMed, EMBASE, Web of Science, OvidSP (Medline), The Cochrane Library and Google Scholar. Studies investigating the diagnostic accuracy of myocardial perfusion imaging (MPI) SPECT in patients evaluated for kidney transplantation were retrieved. After a risk of bias assessment using QUADAS-2, a meta-analysis was conducted.

Results. Out of 1459 records, 13 MPI SPECT studies were included in the meta-analysis with a total of 1245 MPI SPECT scans. There were no studies available with CCTA as reference. Pooled sensitivity of MPI SPECT for CAD was 0.66 (95\% CI 0.53 to 0.77 ), pooled specificity was $0.75(95 \%$ CI 0.63 to 0.84$)$ and the area under the curve (AUC) was 0.76 . Positive likelihood ratio was 2.50 (95\% CI 1.78 to 3.51 ) and negative likelihood ratio was 0.41 (95\% CI 0.28 to 0.61 ). Pooled positive predictive value was $64.9 \%$ and pooled negative predictive value was $74.1 \%$. Significant heterogeneity existed across the included studies.

Conclusions. MPI SPECT had a moderate diagnostic accuracy in patients evaluated for kidney transplantation, with a high rate of false-negative findings. The use of an anatomical

Supplementary Information The online version contains supplementary material available at https://doi.org/10.1007/s12350-02102621-X.

Jeroen R. Kelderman and Floris E. J. Jolink shared first authorship. The authors of this article have provided a PowerPoint file, available for download at SpringerLink, which summarises the contents of the paper and is free for re-use at meetings and presentations. Search for the article DOI on SpringerLink.com.

Funding This study received no funding of any kind.
Reprint requests: Riemer H. J. A. Slart, MD, PhD, University of Groningen, Medical Imaging Centre, Department of Nuclear Medicine and Molecular Imaging, University Medical Center Groningen, Groningen, The Netherlands; r.h.j.a.slart@umcg.nl

J Nucl Cardiol 2022;29:3405-15.

$1071-3581 / \$ 34.00$

Copyright (C) 2021 The Author(s) 
gold standard against a functional imaging test in the included studies is however suboptimal. ( $\mathrm{J}$

Nucl Cardiol 2022;29:3405-15.)

Key Words: End-stage renal disease $\cdot$ Myocardial perfusion imaging • Invasive coronary angiography $\cdot$ Coronary artery disease $\bullet$ Diagnosis $\cdot$ Systematic review $\bullet$ Meta-analysis

\begin{tabular}{|c|c|}
\hline Abbreviation & \\
\hline ICA & Invasive coronary angiography \\
\hline ESRD & End-stage renal disease \\
\hline ESLD & End-stage liver disease \\
\hline CCTA & $\begin{array}{l}\text { Coronary computed tomography } \\
\text { angiography }\end{array}$ \\
\hline SPECT & $\begin{array}{l}\text { Single-photon emission computed } \\
\text { tomography }\end{array}$ \\
\hline MPI & Myocardial perfusion imaging \\
\hline PET & Positron emission tomography \\
\hline CACS & Coronary artery calcium score \\
\hline PLR & Positive likelihood ratio \\
\hline NLR & Negative likelihood ratio \\
\hline DOR & Diagnostic odds ratio \\
\hline AUC & Area under the curve \\
\hline
\end{tabular}

See related editorial, pp. 3416-3418

\section{INTRODUCTION}

Mortality after kidney transplantation has been importantly reduced in the last three decades, particularly in the early post-transplantation period. However, mortality by cardiovascular disease remains an important risk in the first three months after surgery. ${ }^{1}$ This is not unexpected, as reports of invasive coronary angiography (ICA) in patients with end-stage renal disease (ESRD) evaluated for kidney transplantation document a prevalence of coronary artery disease (CAD) between $42 \%$ and $81 \% .^{2}$

In ESRD patients evaluated for kidney transplantation, there is a poor correlation between clinical presentation and significant CAD as only $44 \%$ of dialysis-dependent patients with acute myocardial infarction present with chest pain, compared to $68 \%$ of patients in the general population. ${ }^{3}$ As such, the presentation of CAD is frequently asymptomatic, making it difficult to identify patients that may benefit from medical therapies to reduce the CAD burden. ${ }^{3,4}$ These patients may benefit from cardiovascular screening techniques for asymptomatic $\mathrm{CAD}$ by reducing the procedural risk during a kidney transplantation.

The current gold-standard method for detecting CAD is ICA; however, the invasive nature of this technique makes it only appropriate to perform in the presence of high likelihood of obstructive CAD. An alternative technique is coronary computed tomography angiography (CCTA), a non-invasive method used in patients with low clinical likelihood of obstructive
$\mathrm{CAD}^{2,5}$ Unfortunately, both methods can induce contrast nephropathy in ESRD patients, ${ }^{2,6}$ and CT is often of limited value in ESRD patients due to the high atherosclerotic burden. ${ }^{2}$

In patients with intermediate likelihood of obstructive $\mathrm{CAD}$, there is considerable experience with noninvasive myocardial perfusion imaging (MPI), using single-photon emission computed tomography (SPECT). For the general population, MPI SPECT is considered a reliable diagnostic technique for the functional detection of CAD, as it provides a qualitative and semi-quantitative assessment of the myocardial perfusion defect. ${ }^{7,8}$

We aimed to assess the diagnostic accuracy of SPECT for CAD assessment compared to ICA and CCTA, in patients evaluated for kidney transplantation.

\section{METHODS}

This systematic review and meta-analysis was performed according to the Preferred Reporting Items for Systematic Reviews and Meta-Analyses of Diagnostic Test Accuracy Studies (PRISMA-DTA) statement. ${ }^{9}$ The PRISMA 2009 checklist table is available as a supplement of this article (S1 Appendix). The study protocol is registered with PROSPERO, protocol number CRD42020188610.

\section{Literature Search}

A literature search was performed by two reviewers (JK, FJ) using the following databases: PubMed, EMBASE, Web of Science, OvidSP (Medline), The Cochrane Library and Google Scholar. Detailed search strategies, including Medical Subject Headings (MeSH)terms and Emtree terms, are available as a supplement of this article (S2 Appendix). A medical information specialist examined and verified our search strategies for all the databases. All identified records published until 5th March 2021 were exported.

\section{Selection of Records}

Obtained records were entered into the evidence synthesis tool CADIMA. ${ }^{10}$ After removal of duplicates by CADIMA, the records were independently screened on title and abstract. Randomized controlled trials and observational studies in patients evaluated for kidney transplantation were included if they compared MPI 
SPECT to ICA or CCTA. Papers written in a nonEnglish language, papers where the full text was not available, non-human trials, studies with a small sample size $(n<30)$, conference abstracts, editorials, protocol papers and systematic reviews were excluded. Any inconsistencies were resolved after discussion with an independent third reviewer (SB).

\section{Quality Assessment}

QUADAS-2, a tool developed for the systematic review of diagnostic accuracy studies, was used to assess risk of bias and applicability of a study. ${ }^{11}$ The criteria of the QUADAS-2 tool were: bias due to selection, index test, reference test, and flow and timing. The index tests were MPI and the reference standards were ICA or CCTA. The quality assessment was performed independently by the two reviewers (JK and FJ). Disagreements were resolved by discussing with a third reviewer (SB).

All studies were rated as having a high, low or intermediate/ uncertain risk of bias. Uncertain risk of bias was graded in the following cases: for the domain 'risk of bias due to patient selection' if the studies performed SPECT MPI and ICA only in patients at high risk for $\mathrm{CAD}$, for the domains 'risk of bias due to index test' or 'risk of bias due to reference standard' if it was unsure whether blinding was performed, and for the domain 'risk of bias due to flow and timing' if it was unclear how much time there was between the index test and reference test, and if not all patients underwent both tests.

\section{Data Extraction}

Using a structured template, study characteristics (i.e., design and sample size), baseline population demographics (i.e., age, sex, diabetes mellitus), used definitions for abnormal cardiac testing, and the reported outcomes of individual studies were extracted.

\section{Statistical Methods}

For the diagnostic studies, sensitivity and specificity forest plots were created in RevMan, Version 5.4 (The Cochrane Collaboration, 2020, Copenhagen, Denmark). A bivariate analysis and receiver operating characteristic (ROC) curve were created for the summary estimate and the corresponding $95 \% \mathrm{CI}$ with $\mathrm{R}$ software package: A Language and Environment for Statistical Computing, version 1.0.153 for Mac (R Foundation for Statistical Computing, Vienna, Austria). Heterogeneity was visually checked in forest plots and the summary receiver operating curve (SROC). An $I^{2}$ higher than $50 \%$ was considered indicative of significant study heterogeneity. ${ }^{12}$ Negative and positive likelihood ratios, as well as diagnostic odds ratios (DOR), were calculated with Open Meta-Analyst (OpenMeta [Analyst], Brown School of Public Health, Providence, USA).

\section{RESULTS}

\section{Studies Included}

After duplicate removal, we identified 1459 studies, published between 1990 and 2019. After screening, 13 studies were eligible for qualitative analysis, all targeting MPI SPECT. No studies could be included using the reference standard CCTA (Fig. 1). Ten studies were prospective and three were retrospective. We excluded one study after full-text screening because of suspected data overlap with the study of Winther et al. ${ }^{13}$

\section{Study Characteristics}

A total of 1245 MPI SPECT scans and 1258 ICAs were performed. MPI SPECT images were labeled as abnormal based on the presence of fixed or reversible perfusion defects or the calculated summed stress score. ICA results were labeled as abnormal and a CAD diagnosis was made based on the percentage of stenosis. Five studies used $>50 \%$ stenosis as the cut-off, six studies used $>70 \%$ stenosis or more as the cut-off and two studies used a combination of $>50 \%$ and $>70 \%$ stenosis to determine CAD. The study by Wilson et al. ${ }^{14}$ used $>50 \%$ for the left main coronary artery, $>70 \%$ for epicardial coronary arteries and $50 \%$ to $70 \%$ in borderline lesions. The study by Doukky et al. ${ }^{15}$ used $>50 \%$ for the left main coronary artery and $>70 \%$ stenosis in any of the epicardial coronary arteries (Table 1).

\section{Quality ASSESSMENT}

The results of the QUADAS-2 tool are summarized in Table 2 .

\section{Technical Aspect}

Three different radiopharmaceuticals were used in the studies: Technetium-99m-sestamibi, Technetium99m-tetrofosmin and Thallium-201. All studies performed imaging on conventional Anger gamma camera systems. MPI SPECT/CT imaging was not used, the included studies used MPI SPECT only. The software programs used in the included studies were not mentioned. 


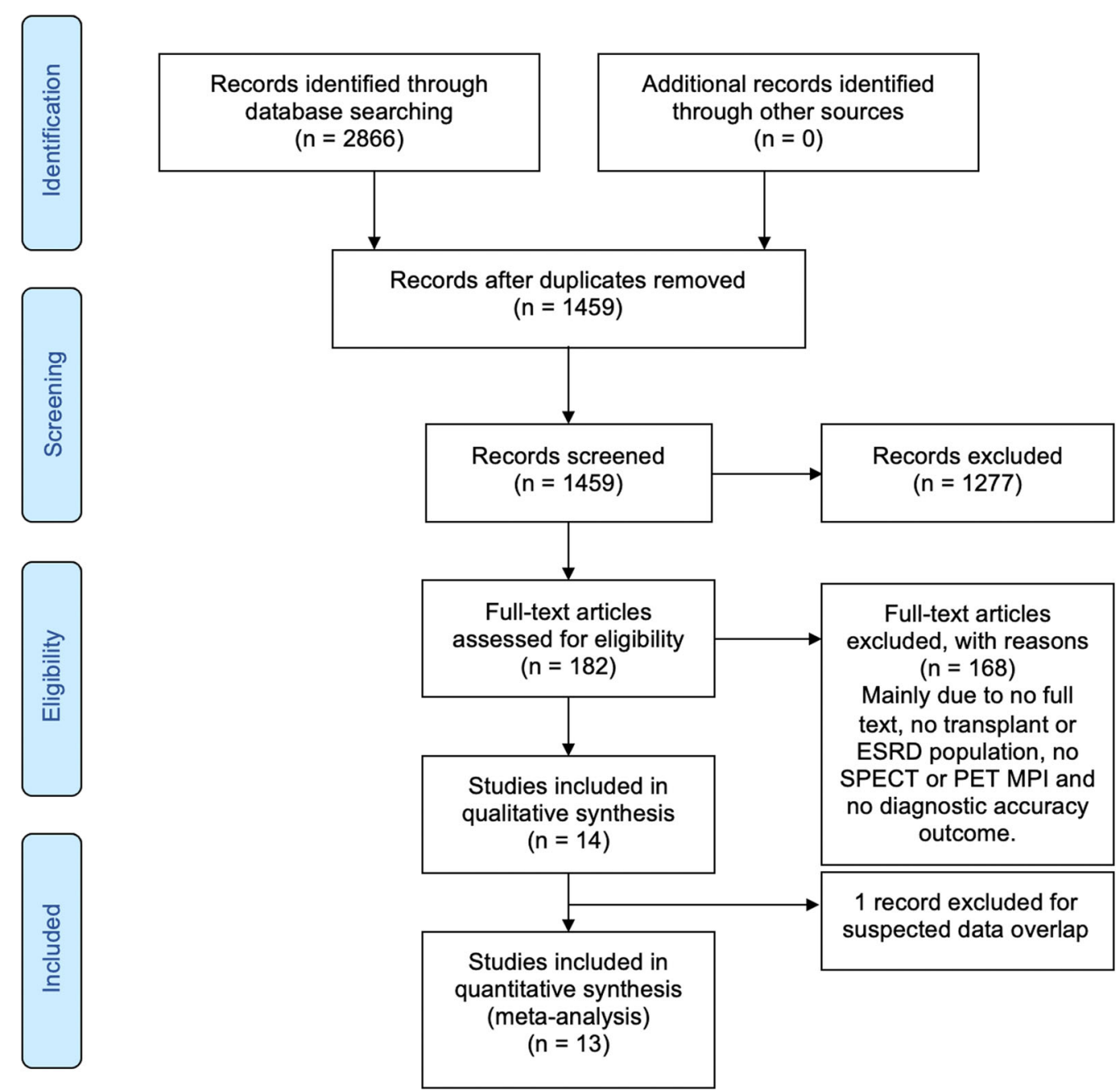

Fig. 1. Flowchart of included studies.

\section{Diagnostic Accuracy}

Diagnostic data were extracted from all included studies $(n=13)$. Three studies performed MPI SPECT as part of standard care, ten studies only in high-risk patients. Sensitivity of the included studies ranged from $34 \%$ to $93 \%$ and specificities ranged from $24 \%$ to $96 \%$ (Fig. 2).

After bivariate analysis a mean sensitivity for MPI SPECT of 0.66 (95\% CI 0.53 to 0.77 ), mean specificity of 0.75 (95\% CI 0.63 to 0.84 ) and an area under the curve (AUC) of 0.76 (Fig. 3) was calculated. Positive likelihood ratio (PLR) was 2.50 (95\% CI 1.78 to 3.51 ) and negative likelihood ratio (NLR) was $0.41(95 \% \mathrm{CI}$ 0.28 to 0.61 ) with an overall diagnostic odds ratio (DOR) of 6.03 (95\% CI 3.46 to 10.50) (Figs. 4, 5, 6). Pooled positive predictive value was $64.9 \%$, pooled negative predictive value was $74.1 \%$, and pooled CAD prevalence was $42.4 \%$. There was evidence of heterogeneity present in the forest plots for sensitivity and specificity (Figs. 2, 4, 3). We found significant heterogeneity $\left(I^{2}>50 \%\right)$ for PLR, NLR, and DOR (Figs. 4, 5, 6).

\section{DISCUSSION}

This systematic review and meta-analysis evaluated the ability of MPI SPECT to detect CAD in patients evaluated for kidney transplantation. With data from 13 studies and a combined total of 1245 MPI SPECT scans, we demonstrated that MPI SPECT had only moderate sensitivity and specificity, ranging between $34 \%$ and $93 \%$ and $24 \%$ to $96 \%$, respectively, for detecting clinically relevant CAD. A high heterogeneity existed across the included studies and there were no studies available with CCTA as reference. 


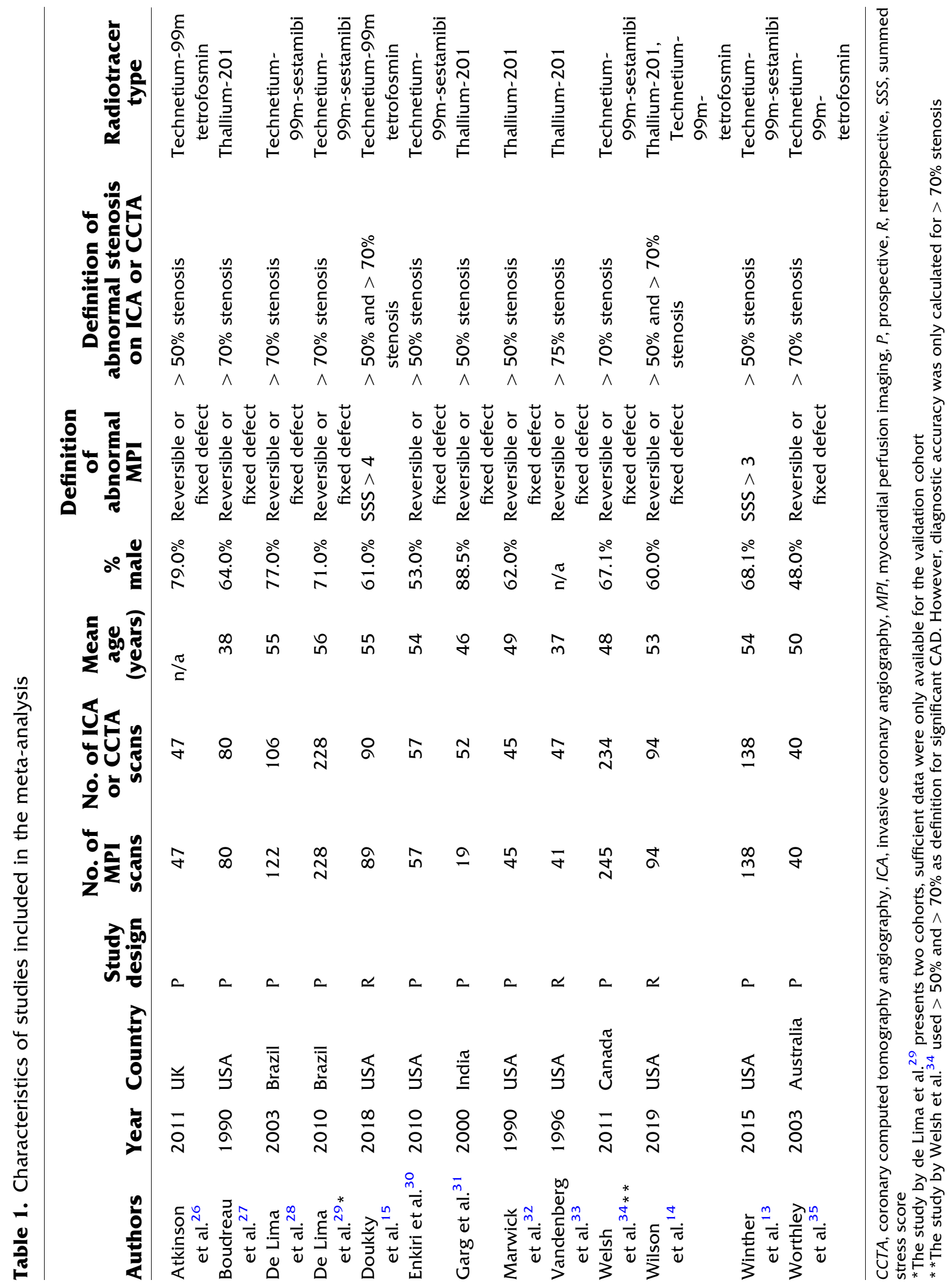




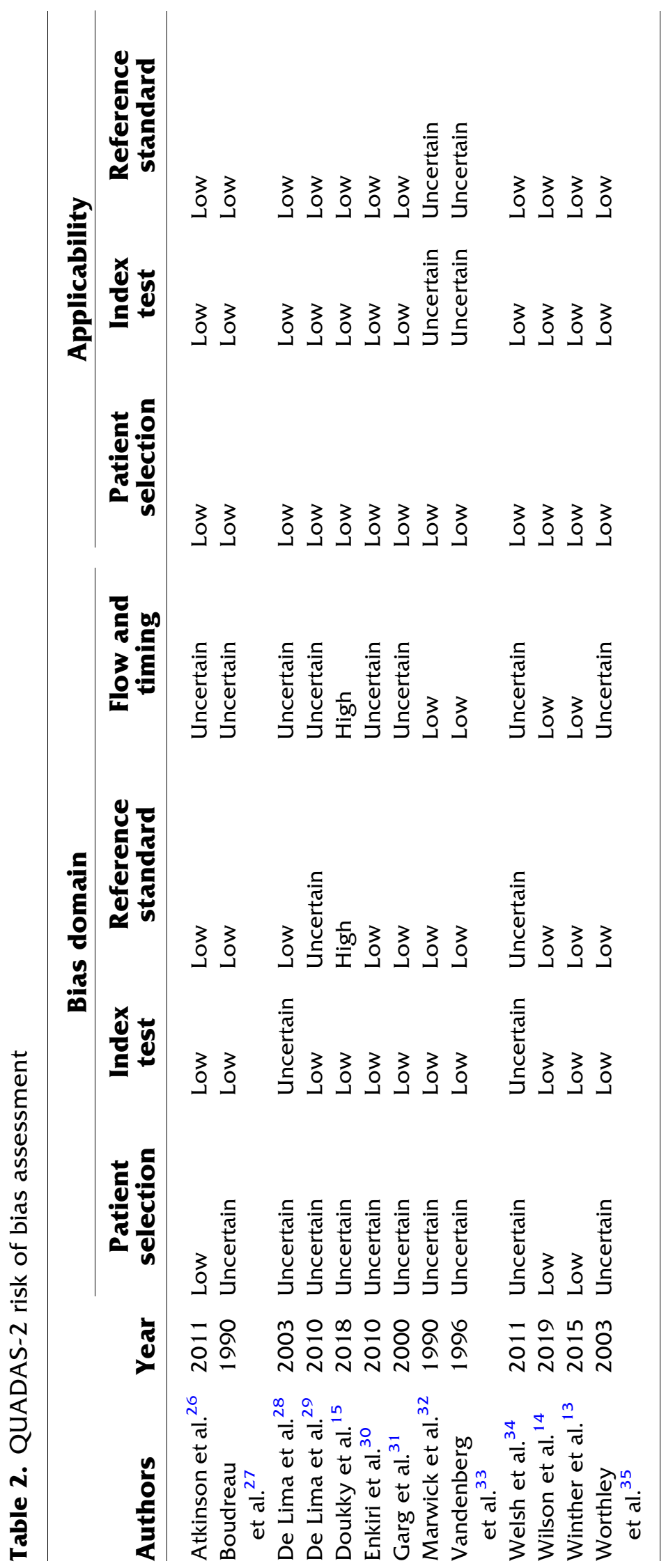




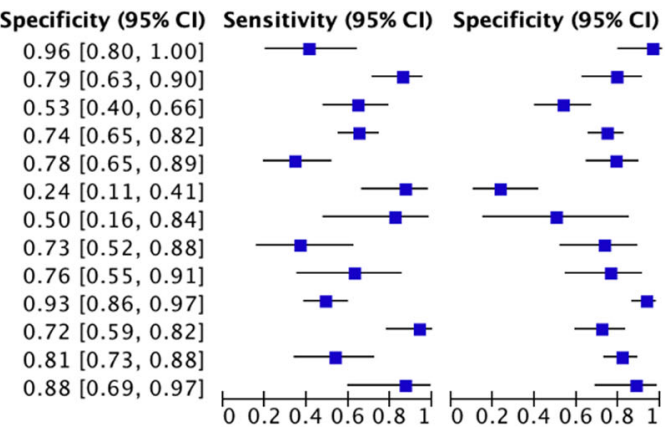

Fig. 2. Sensitivity and specificity of myocardial perfusion imaging SPECT for coronary artery disease in patients evaluated for kidney transplantation.

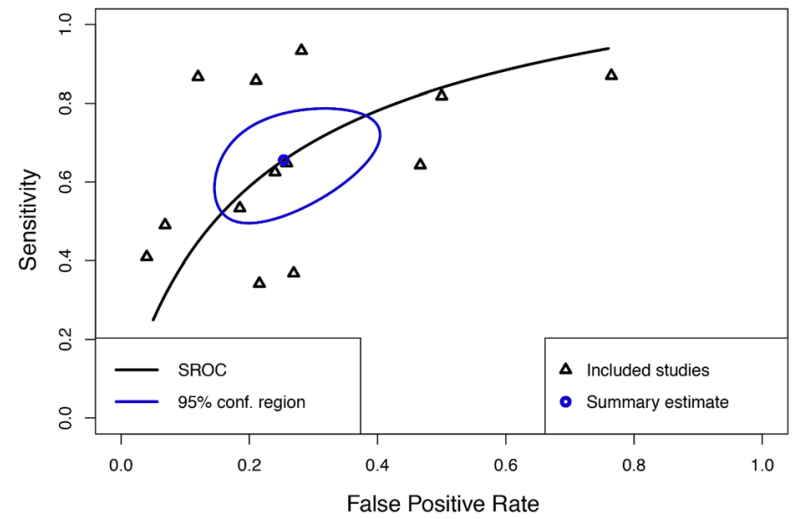

Fig. 3. Summary Receiver Operating Curve (SROC) of included studies.

Although the available studies do not show a high accuracy of MPI SPECT for the diagnose of CAD, current guidelines recommend screening with MPI SPECT in patients with cardiovascular risk factors. ${ }^{16,17}$ A joined statement by the international cardiology and transplantation societies on cardiac disease evaluation in kidney and liver transplantation candidates, recommends non-invasive stress testing when three or more CAD risk factors are present (diabetes mellitus, prior cardiovascular disease, duration of dialysis of $>1$ year, left ventricular hypertrophy, age $>60$ years, smoking, hypertension and dyslipidemia), regardless of functional status (Class IIb, Level of Evidence C). ${ }^{17}$

A meta-analysis on the diagnostic accuracy of MPI SPECT for the detection of CAD in the general population reported a pooled sensitivity and specificity of, respectively, 0.88 (95\% CI 0.88 to 89 ) and 0.61 (95\% CI 0.59 to 0.62$).{ }^{18} \mathrm{~A}$ previous systematic review by Wang et al., determined the diagnostic accuracy of MPI SPECT in ESRD patients. Their study showed a pooled sensitivity of 0.74 (95\% CI 0.54 to 0.87 ) and specificity of 0.70 (95\% CI 0.51 to 0.84$),{ }^{19}$ which is comparable to the outcomes presented in the current study. When comparing MPI SPECT in patients with ESRD to the general population, a lower sensitivity but high specificity is observed. Patients with ESRD often have hypertension, left ventricular hypertrophy and decreased coronary flow reserve, all of which may account for reduced sensitivity of MPI SPECT in kidney transplant candidates, and may explain the differences with the general population. ${ }^{20}$ In patients with end-stage liver disease (ESLD), a meta-analysis compared the diagnostic accuracy of MPI SPECT with adenosine and regadenoson as vasodilating agents. This study reported a pooled sensitivity of 0.62 (95\% CI 0.44 to 0.79$)$ and pooled specificity of 0.82 (95\% CI 0.77 to 0.87 ) for detecting severe CAD with adenosine scans. For regadenoson, these numbers were, respectively, 0.35 ( $95 \%$ CI 0.14 to 0.62 ) and $0.88(0.82$ to 0.92$){ }^{21}$ The sensitivity of MPI SPECT in ESLD patients may be lower than in the general population due to hemodynamic changes and reduced coronary vascular resistance. $^{22}$ Therefore, there might be an impaired response to agents inducing pharmacological stress. The pooled sensitivity and specificity of MPI SPECT for CAD reported in the current study (0.66 and 0.75, respectively) is comparable to the results in patients with ESRD and ESLD, with a lower sensitivity and higher specificity compared to MPI in the general population. Several studies demonstrated the association of perfusion defects on MPI SPECT and cardiovascular events following kidney transplantation. In an analysis of 1189 renal transplant patients, of which 819 underwent MPI SPECT, Ives et al. reported that abnormal MPI SPECT findings is an independent risk factor of cardiovascular events after renal transplantation (Hazard Ratio 1.78 (95\% CI (1.03 to 3.06)). ${ }^{23}$ In a retrospective cohort study with 401 patients who underwent MPI SPECT prior to 


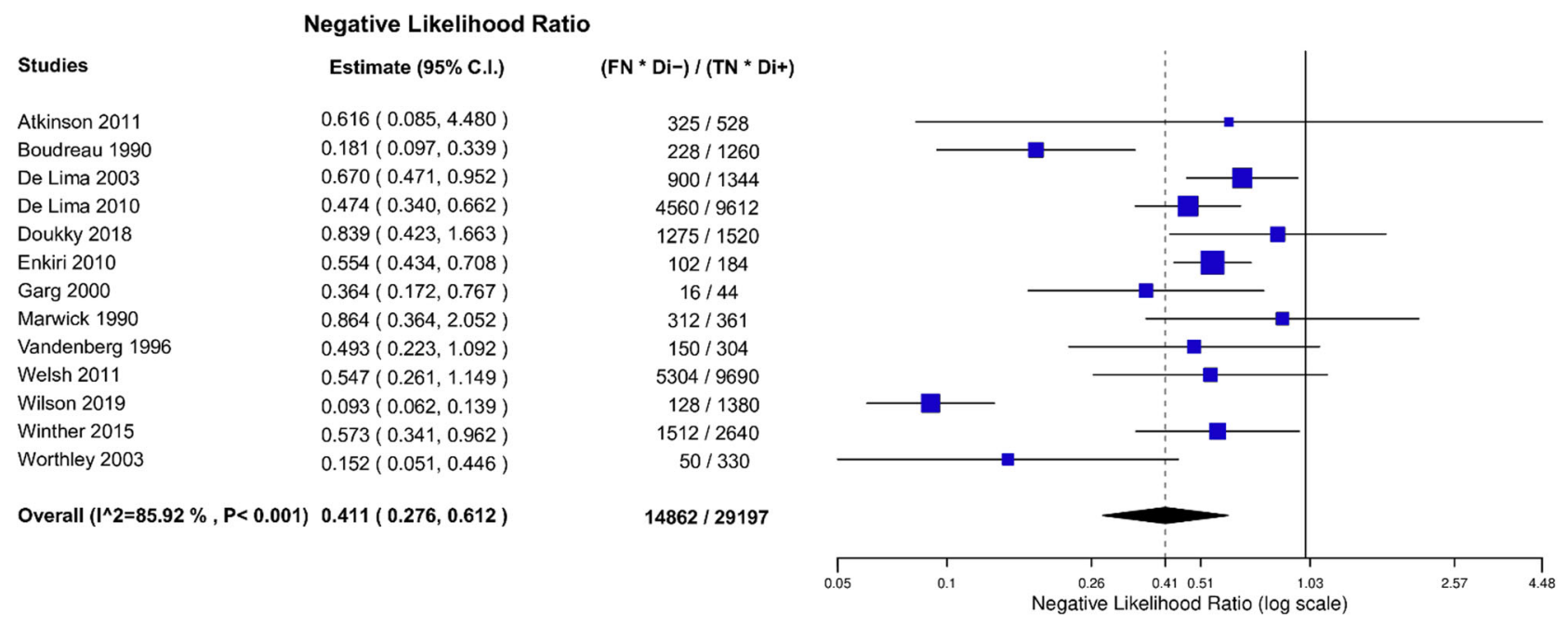

Fig. 4. Negative Likelihood Ratio (NLR) of included studies.

\begin{tabular}{lll} 
Studies & \multicolumn{3}{c}{ Estimate $(\mathbf{9 5} \%$ C.I.) } \\
Atkinson 2011 & \multicolumn{1}{c}{10.227} & $(1.405,74.441)$ \\
Boudreau 1990 & 4.071 & $(2.173,7.629)$ \\
De Lima 2003 & 1.378 & $(0.969,1.959)$ \\
De Lima 2010 & 2.509 & $(1.797,3.502)$ \\
Doukky 2018 & 1.586 & $(0.800,3.144)$ \\
Enkiri 2010 & 1.137 & $(0.890,1.452)$ \\
Garg 2000 & 1.636 & $(0.775,3.453)$ \\
Marwick 1990 & 1.368 & $(0.576,3.249)$ \\
Vandenberg 1996 & 2.604 & $(1.177,5.762)$ \\
Welsh 2011 & 7.143 & $(3.402,14.998)$ \\
Wilson 2019 & 3.319 & $(2.217,4.966)$ \\
Winther 2015 & 2.880 & $(1.715,4.836)$ \\
Worthley 2003 & 7.222 & $(2.453,21.265)$ \\
& & \\
Overall (I^2=80.18 \% , P< 0.001) & $\mathbf{2 . 5 0 0}$ & $(\mathbf{1 . 7 8 1}, \mathbf{3 . 5 0 8})$
\end{tabular}

(TP * Di-) / (FP * Di+)
$225 / 22$
$1368 / 336$
$1620 / 1176$
$8400 / 3348$
$663 / 418$
$680 / 598$
$72 / 44$
$182 / 133$
$250 / 96$
$5100 / 714$
$1792 / 540$
$1728 / 600$
$325 / 45$
$22405 / 8070$

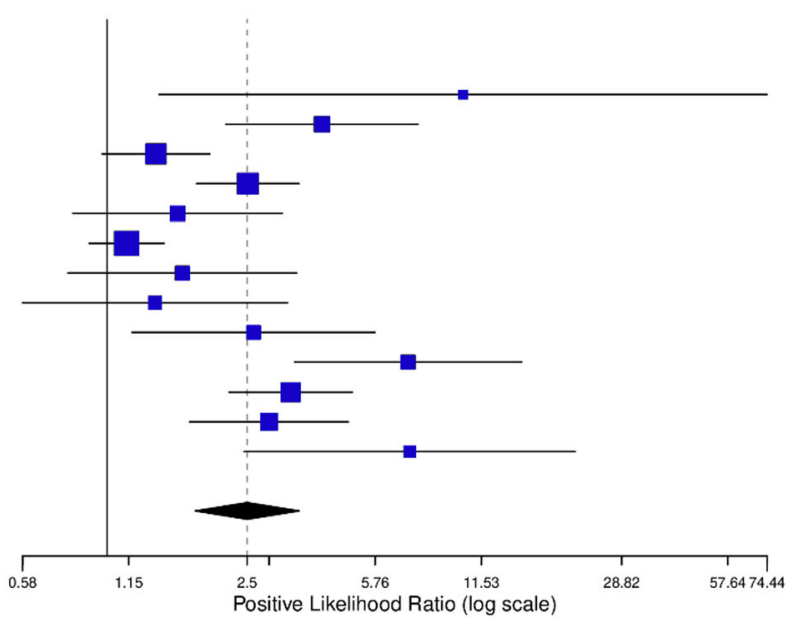

Fig. 5. Positive Likelihood Ratio (PLR) of included studies.

kidney transplantation, Doukky et al. reported that MPI SPECT had long-term prognostic value in patients with 3 or more AHA/ACCF risk factors. ${ }^{15}$ Therefore, MPI SPECT can be used as well as a screening tool to predict cardiovascular events in patients evaluated for renal transplant.

The current review demonstrated that all of the 13 included studies applied visual/semi-quantitative scoring, which resulted in significant heterogeneity across the studies. In the included studies, there was a lack of a standard SPECT procedure, such as a wide variety of radiopharmaceuticals, one- or two-day stress protocols, different types of specialists who assess the imaging findings, differences in pharmacological stress inducers, different software programs, and variation in time interval between injection and MPI SPECT. This may well explain the wide confidence intervals found for sensitivities, specificities, PLR and NLR. This underlines that a standardization of the protocols is warranted which will improve the quality and reproducibility of MPI SPECT. Thus currently the procedural guidelines for cardiac MPI SPECT issued by the European Association of Nuclear Medicine (EANM) are recommended. ${ }^{24}$ 


\section{Diagnostic Odds Ratio}

Studies

Atkinson 2011

Boudreau 1990

De Lima 2003

De Lima 2010

Doukky 2018

Enkiri 2010

Garg 2000

Marwick 1990

Vandenberg 1996

Welsh 2011

Wilson 2019

Winther 2015

Worthley 2003
Estimate (95\% C.I.)

$16.615(1.891,146.024)$

$22.500(7.024,72.070)$

$2.057(0.915,4.623)$

$5.289(2.996,9.337)$

$1.891 \quad(0.734,4.869)$

$2.051 \quad(0.482,8.739)$

$4.500 \quad(0.570,35.519)$

$1.583(0.443,5.653)$

$5.278 \quad(1.346,20.693)$

$13.049(5.521,30.844)$

$35.778(7.712,165.982)$

$5.029 \quad(2.115,11.957)$

$47.667(7.016,323.857)$

Overall $\left(I^{\wedge} 2=67.70 \%, P<0.001\right) 6.027 \quad(3.461,10.496)$

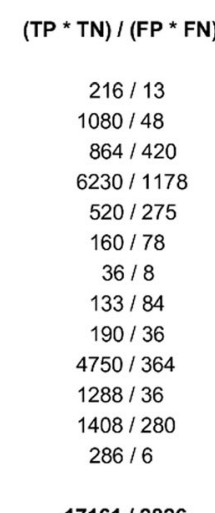

$17161 / 2826$

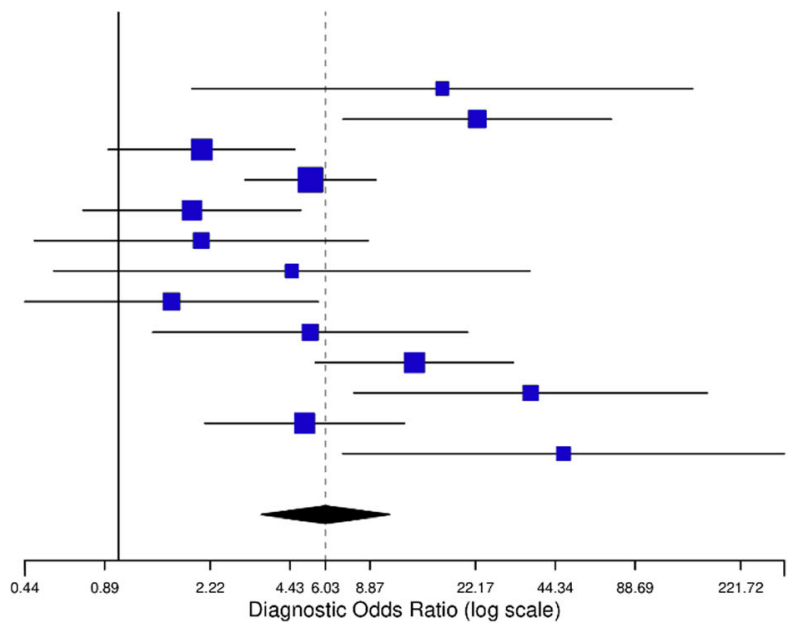

Fig. 6. Diagnostic Odds Ratio (DOR) of included studies.

MPI PET has several advantages compared to MPI SPECT. Due to the routine correction of radiotracer attenuation, the higher spatial resolution, the higher extraction of PET perfusion tracers, PET scanning has a better diagnostic accuracy than SPECT. ${ }^{25}$ The lower radiation burden and the ability to make an absolute quantitative perfusion assessment with PET is another advantage of this method. ${ }^{25}$ However, PET scanning is relative more expensive and less widely available. Production of the short-lived radiopharmaceuticals requires a costly cyclotron or a generator. The diagnostic accuracy of PET has to date not been investigated in the ESRD population although a higher accuracy is expected for the functional diagnosis of CAD when using MPI PET in these patients.

Our study has some limitations that need to be addressed, including those inherent to systematic reviews and meta-analyses of diagnostic test accuracy studies. We included studies before the year 2000 and as with technology, radioisotopes and MPI SPECT procedures have changed and improved over time. This may have influenced the results of the included studies.

MPI SPECT is used to assess the functional significance of CAD and for risk stratification before renal transplantation. On the contrary, the reference standard ICA uses anatomy to assess CAD. An equivalent comparison between MPI SPECT and ICA has its limitations. Selecting $0.89 \%$ of the published studies on the subject since 1990 may have potential bias of including highly selected centers. Strengths of this work are the extensive literature search in the six most recognized databases, reporting according to the PRISMA-DTA statement, ${ }^{9}$ study protocol registration with PROSPERO and a structured and validated bias assessment using the QUADAS-2 tool. ${ }^{11}$

\section{CONCLUSION}

This systematic review and meta-analysis demonstrated that MPI SPECT had a moderate diagnostic accuracy in patients evaluated for kidney transplantation and resulted in a high rate of false-negative findings. The use of an anatomical gold standard against a functional imaging test is however suboptimal. Further research is essential to establish the role of standardized MPI SPECT for the evaluation of patients prior to transplantation, with special attention for the new dynamic MPI SPECT implementation or replacement by MPI PET.

\section{NEW KNOWLEDGE GAINED}

Application of MPI SPECT to detect CAD in patients evaluated for kidney transplantation has been performed. According to our study results MPI SPECT had a moderate diagnostic accuracy for functional CAD in patients evaluated for kidney transplantation as compared to a suboptimal anatomical standard and offers room for technical imaging improvements.

\section{Disclosure}

The authors of this manuscript have no conflicts of interest to disclose.

\section{Open Access}

This article is licensed under a Creative Commons Attribution 4.0 International License, which permits use, 
sharing, adaptation, distribution and reproduction in any medium or format, as long as you give appropriate credit to the original author(s) and the source, provide a link to the Creative Commons licence, and indicate if changes were made. The images or other third party material in this article are included in the article's Creative Commons licence, unless indicated otherwise in a credit line to the material. If material is not included in the article's Creative Commons licence and your intended use is not permitted by statutory regulation or exceeds the permitted use, you will need to obtain permission directly from the copyright holder. To view a copy of this licence, visit http://creativecommons.org/licenses/by/4.0/.

\section{References}

1. Ying T, Shi B, Kelly PJ, Pilmore H, Clayton PA, Chadban SJ. Death after kidney transplantation: An analysis by era and time post-transplant. J Am Soc Nephrol 2020;31:2887-99.

2. Lentine KL, Hurst FP, Jindal RM, Villines TC, Kunz JS, Yuan $\mathrm{CM}$, et al. Cardiovascular risk assessment among potential kidney transplant candidates: Approaches and controversies. Am J Kidney Dis 2010;55:152-67.

3. Sarnak MJ, Amann K, Bangalore S, Cavalcante JL, Charytan DM, Craig JC, et al. Chronic kidney disease and coronary artery disease. J Am Coll Cardiol 2019;74:1823-38.

4. Fujii H, Kono K, Nishi S. Characteristics of coronary artery disease in chronic kidney disease. Clin Exp Nephrol 2019;23:725-32.

5. Knuuti J, Wijns W, Saraste A, Capodanno D, Barbato E, FunckBrentano C, et al. 2019 ESC Guidelines for the diagnosis and management of chronic coronary syndromes: The Task Force for the diagnosis and management of chronic coronary syndromes of the European Society of Cardiology (ESC). Eur Heart J 2019;41:407-77.

6. Cheungpasitporn W, Thongprayoon C, Mao MA, Mao SA, D'Costa MR, Kittanamongkolchai W, et al. Contrast-induced acute kidney injury in kidney transplant recipients: A systematic review and meta-analysis. World J Transpl 2017;7:81-7.

7. Mowatt G, Brazzelli M, Gemmell H, Hillis GS, Metcalfe M, Vale L. Systematic review of the prognostic effectiveness of SPECT myocardial perfusion scintigraphy in patients with suspected or known coronary artery disease and following myocardial infarction. Nucl Med Commun 2005;26:217-29.

8. Mangla A, Oliveros E, Williams KA, Kalra DK. Cardiac imaging in the diagnosis of coronary artery disease. Curr Probl Cardiol 2017;42:316-66

9. McInnes MDF, Moher D, Thombs BD, McGrath TA, Bossuyt PM Group atP-D. Preferred reporting items for a systematic review and meta-analysis of diagnostic test accuracy studies: The PRISMA-DTA statement. JAMA 2018;319:388-96.

10. Kohl C, McIntosh EJ, Unger S, Haddaway NR, Kecke S, Schiemann J, et al. Online tools supporting the conduct and reporting of systematic reviews and systematic maps: A case study on CADIMA and review of existing tools. Environ Evid 2018;7:8.

11. Whiting PF, Rutjes AW, Westwood ME, Mallett S, Deeks JJ, Reitsma JB, et al. QUADAS-2: A revised tool for the quality assessment of diagnostic accuracy studies. Ann Intern Med 2011;155:529-36.

12. Higgins JP, Thompson SG, Deeks JJ, Altman DG. Measuring inconsistency in meta-analyses. BMJ 2003;327:557-60.
13. Winther S, Svensson M, Jørgensen HMS, Bouchelouche K, Gormsen LC, Holm NR et al. Diagnostic performance of coronary computed tomography angiography and myocardial perfusion imaging in kidney transplantation candidates. JACC Cardiovascular Imaging 2015;8.

14. Wilson RS, Lin T, Chambers CE, Kadry Z, Jain AB. Assessing cardiovascular risk in the prerenal transplant population: Comparison of myocardial perfusion imaging and coronary angiography with risk factor stratification. Clin Transplant 2019;33:e13735.

15. Doukky R, Fughhi I, Campagnoli T, Wassouf M, Kharouta M, Vij A, et al. Validation of a clinical pathway to assess asymptomatic renal transplant candidates using myocardial perfusion imaging. $\mathbf{J}$ Nucl Cardiol 2018;25:2058-68.

16. Chadban SJ, Ahn C, Axelrod DA, Foster BJ, Kasiske BL, Kher V, et al. KDIGO clinical practice guideline on the evaluation and management of candidates for kidney transplantation. Transplantation 2020;104:S11-103

17. Lentine KL, Costa SP, Weir MR, Robb JF, Fleisher LA, Kasiske $\mathrm{BL}$, et al. Cardiac disease evaluation and management among kidney and liver transplantation candidates. Circulation 2012;126:617-63.

18. Jaarsma C, Leiner T, Bekkers SC, Crijns HJ, Wildberger JE, Nagel $\mathrm{E}$, et al. Diagnostic performance of noninvasive myocardial perfusion imaging using single-photon emission computed tomography, cardiac magnetic resonance, and positron emission tomography imaging for the detection of obstructive coronary artery disease: A meta-analysis. J Am Coll Cardiol 2012;59:171928.

19. Wang LW, Fahim MA, Hayen A, Mitchell RL, Baines L, Lord S, et al. Cardiac testing for coronary artery disease in potential kidney transplant recipients. Cochrane Database Syst Rev 2011;2011:CD008691-CD.

20. Houghton JL, Frank MJ, Carr AA, von Dohlen TW, Prisant LM. Relations among impaired coronary flow reserve, left ventricular hypertrophy and thallium perfusion defects in hypertensive patients without obstructive coronary artery disease. J Am Coll Cardiol 1990;15:43-51.

21. Bhutani S, Tobis J, Gevorgyan R, Sinha A, Suh W, Honda HM, et al. Accuracy of stress myocardial perfusion imaging to diagnose coronary artery disease in end stage liver disease patients. Am J Cardiol 2013;111:1057-61.

22. Møller S, Bendtsen F. The pathophysiology of arterial vasodilatation and hyperdynamic circulation in cirrhosis. Liver Int 2018;38:570-80.

23. Ives CW, AlJaroudi WA, Kumar V, Farag A, Rizk DV, Oparil S, et al. Prognostic value of myocardial perfusion imaging performed pre-renal transplantation: Post-transplantation follow-up and outcomes. Eur J Nucl Med Mol Imaging 2018;45:1998-2008.

24. Verberne HJ, Acampa W, Anagnostopoulos C, Ballinger J, Bengel $\mathrm{F}$, De Bondt $\mathrm{P}$, et al. EANM procedural guidelines for radionuclide myocardial perfusion imaging with SPECT and SPECT/CT: 2015 revision. Eur J Nucl Med Mol Imaging 2015;42:1929-40.

25. Knaapen P, de Haan S, Hoekstra OS, Halbmeijer R, Appelman YE, Groothuis JG, et al. Cardiac PET-CT: Advanced hybrid imaging for the detection of coronary artery disease. Neth Heart $\mathbf{J}$ 2010;18:90-8

26. Atkinson P, Chiu DY, Sharma R, Kalra PR, Ward C, Foley RN, et al. Predictive value of myocardial and coronary imaging in the long-term outcome of potential renal transplant recipients. Int J Cardiol 2011;146:191-6.

27. Boudreau RJ, Strony JT, duCret RP, Kuni CC, Wang Y, Wilson $\mathrm{RF}$, et al. Perfusion thallium imaging of type I diabetes patients 
with end stage renal disease: Comparison of oral and intravenous dipyridamole administration. Radiology 1990;175:103-5.

28. De Lima JJG, Sabbaga E, Vieira MLC, De Paula FJ, Ianhez LE, Krieger EM, et al. Coronary angiography is the best predictor of events in renal transplant candidates compared with noninvasive testing. Hypertension 2003;42:263-8.

29. De Lima JJ, Wolff Gowdak LH, de Paula FJ, Ianhez LE, Franchini Ramires JA, Krieger EM. Validation of a strategy to diagnose coronary artery disease and predict cardiac events in high-risk renal transplant candidates. Coronary Artery Dis 2010;21:164-7.

30. Enkiri SA, Taylor AM, Keeley EC, Lipson LC, Gimple LW, Ragosta M. Coronary angiography is a better predictor of mortality than noninvasive testing in patients evaluated for renal transplantation. Catheter Cardiovasc Interv 2010;76:795-801.

31. Garg N, Kapoor A, Umesan CV, Sharma RK, Sinha N. Role of pretransplant arteriography in diabetic end-stage renal disease. Asian Cardiovasc Thorac Ann 2000;8:150-4.

32. Marwick TH, Steinmuller DR, Underwood DA, Hobbs RE, Go $\mathrm{RT}$, Swift $\mathrm{C}$, et al. Ineffectiveness of dipyridamole SPECT thallium imaging as a screening technique for coronary artery disease in patients with end-stage renal failure. Transplantation 1990;49:100-3.

33. Vandenberg BF, Rossen JD, Grover-Mckay M, Shammas NW, Burns TL, Rezai K. Evaluation of diabetic patients for renal and pancreas transplantation: Noninvasive screening for coronary artery disease using radionuclide methods. Transplantation 1996;62:1230-5.

34. Welsh RC, Cockfield SM, Campbell P, Hervas-Malo M, Gyenes G, Dzavik V. Cardiovascular assessment of diabetic end-stage renal disease patients before renal transplantation. Transplantation 2011;91:213-8.

35. Worthley MI, Unger SA, Mathew TH, Russ GR, Horowitz JD. Usefulness of tachycardic-stress perfusion imaging to predict coronary artery disease in high-risk patients with chronic renal failure. Am J Cardiol 2003;92:1318-20.

Publisher's Note Springer Nature remains neutral with regard to jurisdictional claims in published maps and institutional affiliations. 